\title{
Deep Learning Framework for Multi-class Breast Cancer Histology Image Classification
}

\author{
Yeeleng S. Vang, Zhen Chen, and Xiaohui Xie \\ University of California Irvine \\ Irvine, CA 92697 \\ \{ysvang, zhenc4\}@uci.edu xhx@ics.uci.edu
}

\begin{abstract}
In this work, we present a deep learning framework for multiclass breast cancer image classification as our submission to the International Conference on Image Analysis and Recognition (ICIAR) 2018 Grand Challenge on BreAst Cancer Histology images (BACH). As these histology images are too large to fit into GPU memory, we first propose using Inception V3 to perform patch level classification. The patch level predictions are then passed through an ensemble fusion framework involving majority voting, gradient boosting machine (GBM), and logistic regression to obtain the image level prediction. We improve the sensitivity of the Normal and Benign predicted classes by designing a Dual Path Network (DPN) to be used as a feature extractor where these extracted features are further sent to a second layer of ensemble prediction fusion using GBM, logistic regression, and support vector machine (SVM) to refine predictions. Experimental results demonstrate our framework shows a $12.5 \%$ improvement over the state-of-the-art model.
\end{abstract}

\section{Introduction}

In the United States, breast cancer continues to be the leading cause of cancer death among women of all races [1. Studies have shown that improvement to survival rate over the last decade can be attributed to early diagnosis and awareness of better treatment options [2, [3], [4]. Common non-invasive screening test includes clinical breast exam which involves a visual check of the skin and tissue and a manual check for unusual texture or lump, mammography which requires taking an x-ray image of the breast to look for changes, and breast MRI which uses radio waves to obtain a detailed image inside the breast. Of the latter two diagnostic modals, many computer-aided diagnosis (CAD) systems have been developed to assist radiologists in their effort to identify breast cancer in its early stages [5]. On the other side of the screening toolbox are biopsies which are minimally invasive procedures whereby tissue samples are physically removed to be stained with hematoxylin and eosin (H\&E) and visualized under a microscope. These histopathology slides allow pathologists to distinguish between normal, non-malignant, and malignant lesions [11] to assist in their diagnosis. However, even among trained pathologists the concordance between their unanimous agreement is a mere $75 \%$ [6]. This high degree of discord motivates the 
development of automatic CAD systems using machine learning to assist these professionals in their diagnosis.

From November 2017 to January 2018, the International Conference on Image Analysis and Recognition (ICIAR) held the 2018 Grand Challenge on BreAst Cancer Histology images (BACH) to solicit submissions of automatic image analysis systems for the task of four-class classification of breast cancer histology images. Here we present a deep learning framework for the task of multi-class breast cancer histology image classification. Our approach uses the Inception (GoogLeNet) V3 [19] architecture to discriminate between invasive carcinoma, in situ carcinoma, benign lesion, and normal tissue patches. We then fuse these patch level predictions to obtain image level prediction using an ensemble framework. Our system improves the sensitivity over the benign and normal classes by using a Dual Path Network (DPN) [24] to extract features as input into a second level ensemble framework involving GBM, SVM, and logistic regression. Experimental results on a held out set demonstrate our framework shows a $12.5 \%$ improvement over the state-of-the-art model.

\section{Relate Work}

Several works have been published in the area of applying machine learning algorithms for cancer histology image detection and classification [12, 13, ,14, 21]. In the specific area of breast cancer histopathology classification, the Camelyon 16 competition led to numerous new approaches utilizing techniques from deep learning to obtain results comparable to highly trained medical doctors [9, [10, [17. The winning team used Inception V3 to create a tumor probability heatmap and perform geometrical and morphological feature selection over these heatmaps as input into a random forest classifier to achieve near $100 \%$ area under the receiver operating characteristic curve (AUC) score 9. However, this competition involved only binary class prediction of tumor and normal whole slide images. For 4-class breast cancer classification, Araujo et al. [1] published a bespoke convolutional neural network architecture that achieved state-of-theart accuracy results and high sensitivity for carcinoma detection.

\section{ICIAR2018 Grand Challenge Datasets and Evaluation Metric}

In this section, we describe the ICIAR2018 dataset provided by the organizers for the subchallenge of multi-class breast cancer histology image classification and the evaluation metric used to score submissions. The interested reader is encouraged to refer to the competition page for details regarding the other subchallenge.

\subsection{ICIAR2018 Dataset}

The ICIAR2018 breast cancer histology image classification subchallenge consist of Hematoxylin and eosin $(\mathrm{H} \& \mathrm{E})$ stained microscopy images as shown in Table 
1. The dataset is an extended version of the one used by Araujo et al. 11]. All images were digitized with the same acquisition conditions, with resolution of $2040 \times 1536$ pixels and pixel size of $0.42 \mu \mathrm{m} \times 0.42 \mu \mathrm{m}$. Each image is labeled with one of four classes: i) normal tissue, ii) benign lesion, iii) in situ carcinoma and iv) invasive carcinoma according to the predominant cancer type in each image. The images were labeled by two pathologists who only provided a diagnostic from the image contents without specifying the area of interest. There are a total of 400 microscopy images with an even distribution over the four classes. We randomly perform a 70\%-20\%-10\% training-validation-test split. The training and validation sets are used for model development while the test set is held out and only used for evaluation.

Table 1. ICIAR2018 H\&E Histopathology Dataset

\begin{tabular}{c|c|c|c|c|c}
\hline \multicolumn{2}{c|}{ Type } & Training & Validation & Test & Total \\
\hline \multirow{4}{*}{ Microscopy } & normal & 70 & 20 & 10 & \\
& benign & 70 & 20 & 10 & \multirow{2}{*}{400} \\
& in situ & 70 & 20 & 10 & \\
& invasive & 70 & 20 & 10 & \\
\hline
\end{tabular}

\subsection{Evaluation Metric}

This challenge consists of automatically classifying H\&E-stained breast cancer histology images into four classes: normal, benign, in situ carcinoma and invasive carcinoma. Performance on this challenge is evaluated based on the overall prediction accuracy, i.e. the ratio of correct predictions over total number of images.

\section{Method}

In this section, we describe our framework and approach to this problem of multi-class breast cancer histology image classification.

\subsection{Image-wise classification of microscopy images}

Stain Normalization Pre-processing Stain normalization is a critically important step in the pre-processing of $\mathrm{H} \& \mathrm{E}$ stain images. It is known that cell nucleus are stained with a large amount of pure hematoxylin and a small amount of Eosin whereas cytoplasm is stained with a large amount of pure eosin and small amount of hematoxylin [8]. Variations in H\&E images can be attributed to such factors as differences in lab protocols, concentration, source manufacturer, scanners, and even staining time [22]. These variations makes it difficult 
for software trained on a particular stain appearance [23] therefore necessitates careful preprocessing to reduce such variances.

Many methods have been proposed for stain normalization including [23, ,7], 8] that are based on color devolution where RGB pixel values are decomposed into their stain-specific basis vectors. In addition to color information, Bejnordi et el. takes advantage of spatial information to perform this deconvolution step [22, however their approach currently only works for whole slide images.

In our framework, we utilized both Macenko [7, which used singular value decomposition (SVD), and Vahadane normalizations [8, which used sparse nonnegative matrix factorization (SNMF), as part of our ensemble framework. This was due to the fact that initial empirical results showed Macenko-normalized images obtained high sensitivity for invasive and in situ classes whereas Vahadanenormalized images showed high sensitivity for benign and normal classes. Both set of normalized datasets were normalized using "iv001.tif" as the target image. An example of both normalization schemes are shown in Fig. 1.
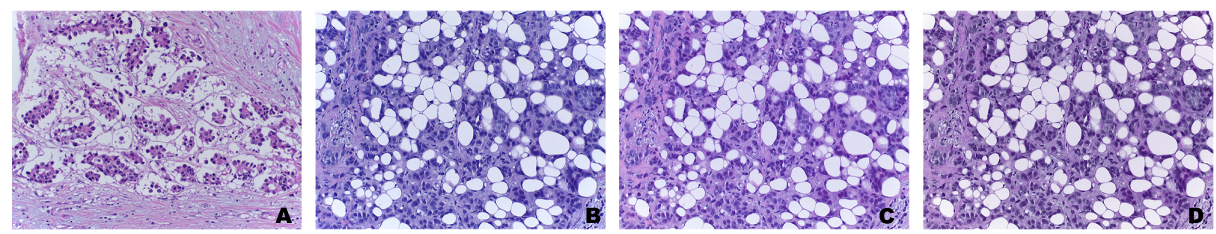

Fig. 1. $A$ target image, $B$ original image, $C$ image after Macenko normalization, $D$ image after Vahadane normaliztion.

Image-wise Classification Framework The microscopy classification framework consists of a patch level classification stage, an image level heatmap postprocessing stage, and possibly a refinement stage, as depicted in Fig. 2. During model training of the patch-based classifer, each patch input is of size $512 \times 512$. We extracted 500 patches from each microscopy slide in the training and validation sets for both Macenko-normalized and Vahadane-normalized datasets. 35 of those patches comes from sliding over the normalized microscopy image with strides of 256 while the remaining patches were randomly sub-sampled. As with the assumption used in [11, these patches are given the same label as the original slide image with which they where obtained from.

A pretrained Inception V3 model [19], is modified to accept image patch of this size and trained to discriminate between the four classes. At training time, images data are dynamically augmented before being fed through the model. Similar to the color perturbation scheme used in 10, brightness is perturbed with a delta of $5 / 255$, contrast with a delta of .05 , saturation with a delta of .05 , and hue with a delta of 0.02 . In addition to color perturbation, images were randomly flipped vertically and/or horizontally, and randomly rotated by 90 degrees to obtain all eight valid orientations. 
The Inception V3 model was fine-tuned on 4 GPUs (2 Nvidia Titan X GPUs and 2 Nvidia GTX 1080Ti) where each GPUs receive a batch of 8 images. Model is trained for 30 epochs with learning rates set as: $5 \mathrm{e}-5$ for the bottom 5 convolution layers, $5 \mathrm{e}-4$ for the eleven inception modules, and $5 \mathrm{e}-2$ for the top fully connected layer. Learning rate was decreased by 0.95 every 2 epochs. The RMSprop optimizer 18 with 0.9 momentum is used and the best performing model on the validation set is saved.

At inference time for a single microscopy image, a heatmap tensor of size $[8 \times 4 \times 3 \times 4]$ is obtained. The first dimension corresponds to the 8 valid orientations of the image, the second dimension to the 4 classes, and the third and fourth dimension corresponds to the spatial dimensions of the image using non-overlapping patching.

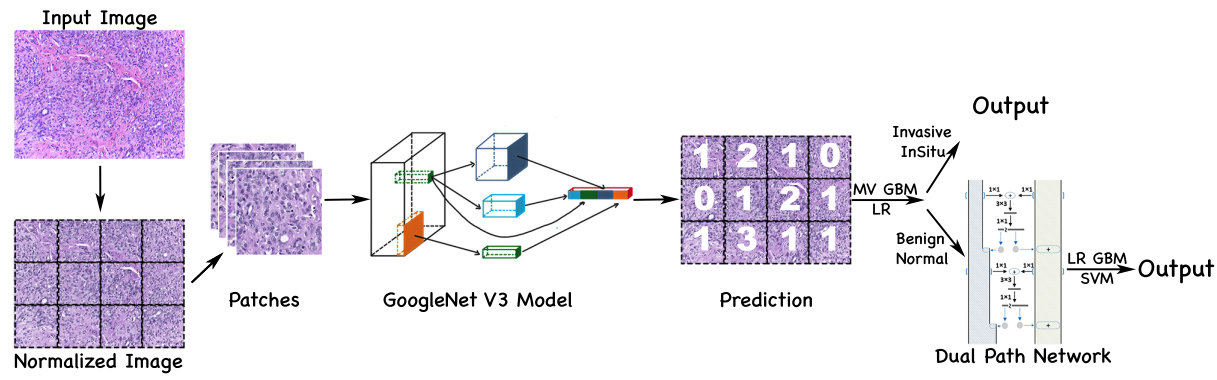

Fig. 2. The framework of image-wise classification. The normalized input image is patched into twelve non-overlapping patches. 8 sets of these patches are generated corresponding to the 8 valid orientations. These 8 sets of patches are passed through the Inception (GoogLeNet) V3 model to generate a patch level heatmap probability tensor. The heatmap tensor is then fused using majority voting (MV), gradient boosting machine (GBM), and logistic regression (LR) across both macenko-normalized and vahadane-normalized version of the input image. If the model predicts invasive or in situ carcinoma, the model outputs this prediction. Otherwise the normalize images are pass through the DPN network to extract features for a second fusing step involving LR, GBM, and support vector machine (SVM) to output prediction for benign and normal class.

Heatmap-based Post-processing Three data fusion strategies were investigated for this competition. The first strategy involved finding the average probabilities along the first dimension of the heatmap and then assigning labels to each $3 \times 4$ patches corresponding to the most probable class, which we will call the class map. From this $3 \times 4$ class map, a final label for the microscopy is obtained by majority voting. The second and third strategies involved finding the class map for each of the 8 orientation separately first, and then obtaining a histogram of the classes across all 8 orientations. The histogram data is then used to train two separate models: a logistic regression with $L 1$ regularization 
and a gradient boosting machine $(\mathrm{GBM}$ ) classifier (num. of estimator $=280$, $\max$ depth $=4$, learning rate $=.9$ ) to ultimately classify the image similar to [16]. If the model predicts benign or normal, the vahadane-normalized image was further passed through a refinement stage as will be describe in the next section.

Refinement model for Benign and Normal classes Since the Inception model yielded low sensitivity for both normal and benign classes with many interclass misclassification between these two classes, we proposed training a slimed-down version of the dual path network (DPN) 24 to serve as a feature extractor for use with Vahadane-normalized images. DPN was chosen due to its compact size and having beneficial characteristics of both residual-like and densenet-like architectures. Using the features extracted by the DPN, we train three additional models: GBM, Support Vector Machine (SVM), and Logistic regression with $L 1$ for binary classification. The results for our entire pipeline is presented below in Table 2

\section{Experimental Results}

The performance of our framework on image-wise classification is shown below in Table 2. As a baseline, we compare against Araujo et al. 11] which, although using a smaller subset of this dataset, tested on a held-out set of roughly the same size. Their best accuracy performance on this 4-class classification problem was $77.8 \%$. Our framework achieves an accuracy score of $87.5 \%$, a $12.5 \%$ improvement over the baseline score. Even without the refinement model, our model offers a $6 \%$ improvement over the baseline.

Table 2. Image-wise Classification Results

\begin{tabular}{|c|c|c|c|}
\hline & \multicolumn{2}{|c|}{ Accuracy } \\
\hline & & Validation Set & Test Set \\
\hline \multirow{3}{*}{$\begin{array}{c}\text { Macenko } \\
\text { normalization }\end{array}$} & MV & 0.800 & 0.775 \\
\hline & LR & 0.750 & 0.775 \\
\hline & GBM & 0.775 & 0.775 \\
\hline \multirow{3}{*}{$\begin{array}{c}\text { Vahadane } \\
\text { normalization }\end{array}$} & MV & 0.788 & 0.775 \\
\hline & LR & 0.763 & 0.775 \\
\hline & GBM & 0.750 & 0.800 \\
\hline \multicolumn{2}{|c|}{ Ensemble } & 0.825 & 0.825 \\
\hline \multicolumn{2}{|c|}{ Ensemble with refinement } & 0.838 & 0.875 \\
\hline
\end{tabular}

Comparing the sensitivity by Araujo et al. [11, we see they achieved sensitivities of $77.8 \%, 66.7 \%, 88.9 \%$, and $88.9 \%$ for normal, benign, in situ, and invasive classes respectively. From Table 3, we showed higher sensitivity across all four classes using our framework. Of noticeable improvement is the benign class which we saw an almost $20 \%$ improvement. This validates our decision 
to incorporate a binary class refinement phase specifically for the benign and normal classes.

Table 3. Image-wise Test Set Contingency Table

\begin{tabular}{|c|c|c|c|c|c|}
\hline Ground Truth $\quad$ Prediction & invasive & in situ & benign & normal & sensitivity \\
\hline invasive & 9 & 0 & 1 & 0 & 0.90 \\
\hline in situ & 0 & 10 & 0 & 0 & 1.00 \\
\hline benign & 1 & 1 & 8 & 0 & 0.80 \\
\hline normal & 0 & 0 & 2 & 8 & 0.80 \\
\hline
\end{tabular}

\section{Discussion}

In this work we proposed a deep learning framework for the problem of multiclass breast cancer histology image classification. To leverage the advances from the computer vision field, we propose using the successful inception V3 model for initial four-class classification. We propose a new ensemble scheme to fuse patch probabilities for image-wise classification. To improve the sensitivity of the benign and normal class, we propose a two-class refinement stage using a dual path network to first extract features from the vahadane-normalized images and then using gradient boosting machine, support vector machine, and logistic regression to fuse all our predictions into a final result. Experimental results on the ICIAR2018 Grand Challenge dataset demonstrates an improvement of $12.5 \%$ over the state-of-the-art system.

\section{References}

1. U.S. Cancer Statistics Working Group. United States Cancer Statistics: 1999-2014 Incidence and Mortality Web-based Report. Atlanta: U.S. Department of Health and Human Services, Centers for Disease Control and Prevention and National Cancer Institute; 2017. Available at: www.cdc.gov/uscs.

2. Saadatmand, Sepideh, et al. "Influence of tumour stage at breast cancer detection on survival in modern times: population based study in 173797 patients." bmj 351 (2015): h4901.

3. Berry, Donald A., et al. "Effect of screening and adjuvant therapy on mortality from breast cancer." New England Journal of Medicine 353.17 (2005): 1784-1792.

4. de Gelder, Rianne, et al. "The effects of populationbased mammography screening starting between age 40 and 50 in the presence of adjuvant systemic therapy." International journal of cancer 137.1 (2015): 165-172.

5. Hadjiiski, Lubomir, Berkman Sahiner, and Heang-Ping Chan. "Advances in CAD for diagnosis of breast cancer." Current opinion in obstetrics \& gynecology 18.1 (2006): 64 . 
6. Elmore, Joann G., et al. "Diagnostic concordance among pathologists interpreting breast biopsy specimens." Jama 313.11 (2015): 1122-1132.

7. Macenko, Marc, et al. "A method for normalizing histology slides for quantitative analysis." Biomedical Imaging: From Nano to Macro, 2009. ISBI'09. IEEE International Symposium on. IEEE, 2009.

8. Vahadane, Abhishek, et al. "Structure-preserved color normalization for histological images." Biomedical Imaging (ISBI), 2015 IEEE 12th International Symposium on. IEEE, 2015.

9. Wang, Dayong, et al. "Deep learning for identifying metastatic breast cancer." arXiv preprint arXiv:1606.05718 (2016).

10. Liu, Yun, et al. "Detecting cancer metastases on gigapixel pathology images." arXiv preprint arXiv:1703.02442 (2017).

11. Arajo, Teresa, et al. "Classification of breast cancer histology images using Convolutional Neural Networks." PloS one 12.6 (2017): e0177544.

12. Nayak, Nandita, et al. "Classification of tumor histopathology via sparse feature learning." Biomedical Imaging (ISBI), 2013 IEEE 10th International Symposium on. IEEE, 2013.

13. Gorelick, Lena, et al. "Prostate histopathology: Learning tissue component histograms for cancer detection and classification." IEEE transactions on medical imaging 32.10 (2013): 1804-1818.

14. $\mathrm{Xu}, \mathrm{Yan}$, et al. "Weakly supervised histopathology cancer image segmentation and classification." Medical image analysis 18.3 (2014): 591-604.

15. Ciompi, Francesco, et al. "The importance of stain normalization in colorectal tissue classification with convolutional networks." arXiv preprint arXiv:1702.05931 (2017).

16. Hou, Le, et al. "Patch-based convolutional neural network for whole slide tissue image classification." Proceedings of the IEEE Conference on Computer Vision and Pattern Recognition. 2016.

17. Bejnordi, Babak Ehteshami, et al. "Diagnostic assessment of deep learning algorithms for detection of lymph node metastases in women with breast cancer." Jama 318.22 (2017): 2199-2210.

18. Tieleman, Tijmen, et al.: Lecture 6.5-rmsprop: Divide the gradient by a running average of its recent magnitude (2012) 22.

19. Szegedy, Christian, Wei Liu, Yangqing Jia, Pierre Sermanet, Scott Reed, Dragomir Anguelov, Dumitru Erhan, Vincent Vanhoucke, and Andrew Rabinovich. "Going deeper with convolutions." In Proceedings of the IEEE conference on computer vision and pattern recognition, pp. 1-9. 2015.

20. Otsu, Nobuyuki. "A threshold selection method from gray-level histograms." IEEE transactions on systems, man, and cybernetics 9, no. 1 (1979): 62-66.

21. Wang, Wei, John A. Ozolek, and Gustavo K. Rohde. "Detection and classification of thyroid follicular lesions based on nuclear structure from histopathology images." Cytometry Part A 77.5 (2010): 485-494.

22. Bejnordi, Babak Ehteshami, et al. "Stain specific standardization of whole-slide histopathological images." IEEE transactions on medical imaging 35.2 (2016): 404415.

23. Khan, Adnan Mujahid, et al. "A nonlinear mapping approach to stain normalization in digital histopathology images using image-specific color deconvolution." IEEE Transactions on Biomedical Engineering 61.6 (2014): 1729-1738.

24. Chen, Yunpeng, et al. "Dual path networks." Advances in Neural Information Processing Systems. 2017. 